\title{
Editorial
}

\section{Cancer Prevention in Low- and Middle-Income Countries}

\author{
Subhojit Dey, ${ }^{1}$ Preet K. Dhillon, ${ }^{2}$ and Preetha Rajaraman ${ }^{3}$ \\ ${ }^{1}$ Indian Institute of Public Health-Delhi, Public Health Foundation of India, Gurgaon, Haryana, India \\ ${ }^{2}$ Centre for Chronic Conditions and Injuries, Public Health Foundation of India, Gurgaon, Haryana, India \\ ${ }^{3}$ Center for Global Health, National Cancer Institute, New Delhi, India \\ Correspondence should be addressed to Subhojit Dey; subhojit.dey@iiphd.org
}

Received 15 January 2017; Accepted 18 January 2017; Published 22 February 2017

Copyright (C) 2017 Subhojit Dey et al. This is an open access article distributed under the Creative Commons Attribution License, which permits unrestricted use, distribution, and reproduction in any medium, provided the original work is properly cited.

Cancer is a rising problem across the world with a $33 \%$ increase in global cases of cancer between 2005 and 2015 [1]. The increase has been maximum in countries with the lowest development [1]. While LMICs bear a major share of the burden of cancer [1-3], very few LMICs have a comprehensive cancer prevention strategy in place. This leads to a high proportion of patients presenting at tertiary care centres at late stages of cancer, when treatment is most difficult and costly and survival is poor [4]. In the absence of adequate treatment capacity in most LMICs, patients presenting at later stages significantly increase the burden of disease. Lack of palliative care [5] compounds this situation further resulting in an unfortunate scenario where a diagnosis of cancer is equated with death in most LMICs. With reducing levels of infectious diseases and rising life expectancies, LMICs are recognizing that cancer needs to become a health priority. However, the essential steps required to prevent cancer and avoid the later consequences are still lacking emphasis. With the above scenario in mind, we had issued a call for papers that focused on cancer prevention in LMICs.

Overall, it was observed that research on cancer and especially cancer prevention in LMICs was limited [6]. Among the cancer research that is being done in LMICs, a lot of research is not of good quality. Also, most of the focus is currently on building capacity and conducting research related to cancer treatment, and cancer prevention takes a back seat, for cancer prevention requires not only facilities and human resources on the health system side but also awareness and the will and ability to pay for preventive services on the population side. Given the situation in most LMICs, both sides fall short of reaching a point where cancer prevention can be a realistic scenario. Regarding this, it is important to note the recommendations of the Breast Health Global Initiative (BHGI) which has created guidelines for breast cancer prevention while considering the economic situation of a particular nation [7]. We need to focus more on cancer prevention in LMICs, and while the job of the health providers and researchers including those who contributed to this special issue is commendable, there is immense scope for more to be done.

Subhojit Dey

Preet K. Dhillon

Preetha Rajaraman

\section{References}

[1] C. Fitzmaurice, C. Allen, R. M. Barber et al., "Global, regional, and national cancer incidence, mortality, years of life lost, years lived with disability, and disability-adjusted life-years for 32 cancer groups, 1990 to 2015: a systematic analysis for the global burden of disease study," JAMA Oncology, 2016.

[2] B. W. Stewart and C. P. Wild, Eds., World Cancer Report, International Association for Research on Cancer (IARC), WHO, 2014.

[3] World Cancer Factsheet, 2014, Cancer Research, 2014, https:// publications.cancerresearchuk.org/downloads/product/CS REPORT_WORLD.pdf.

[4] C. Allemani, H. K. Weir, H. Carreira et al., "Global surveillance of cancer survival 1995-2009: analysis of individual data for 25676887 patients from 279 population-based registries in 67 countries (CONCORD-2)," The Lancet, vol. 385, no. 9972, pp. 977-1010, 2015. 
[5] Cancer Control Opportunities in Low- and Middle-Income Countries, Institute of Medicine (US) Committee on Cancer Control in Low- and Middle-Income Countries, F. A. Sloan, and H. Gelband, Eds., Palliative Care, Cancer Control Opportunities in Low- and Middle-Income Countries, National Academies Press (US), Washington, DC, USA, 2007, https://www.ncbi.nlm .nih.gov/books/NBK54036/.

[6] D. C. Stegan, Ed., Cancer Research and Clinical Trials in Developing Countries: A Practical Guide, Springer, 2015.

[7] M. I. Echavarria, B. O. Anderson, C. Duggan, and B. Thompson, "Global uptake of BHGI guidelines for breast cancer," The Lancet Oncology, vol. 15, no. 13, pp. 1421-1423, 2014. 


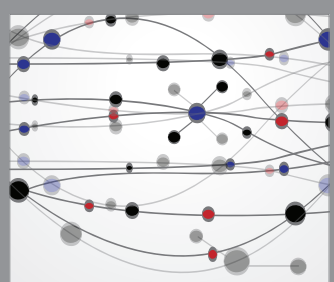

The Scientific World Journal
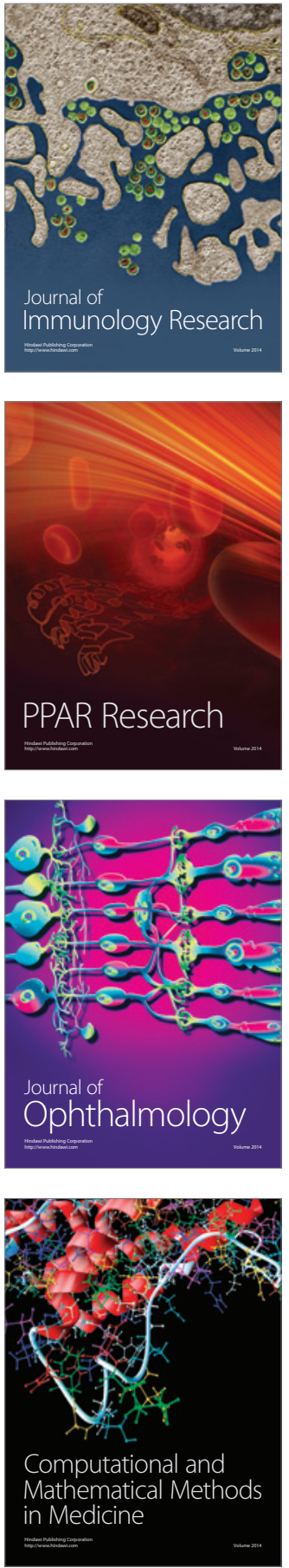

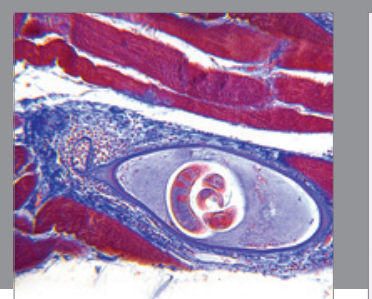

Gastroenterology Research and Practice
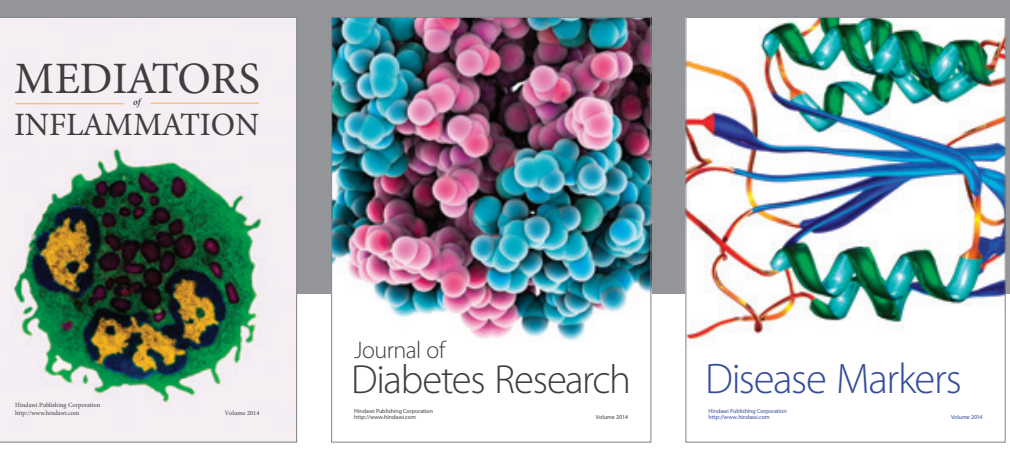

Disease Markers

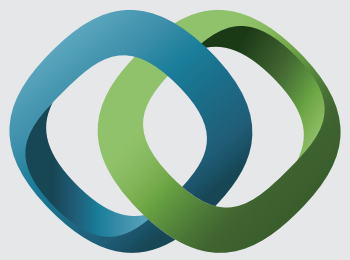

\section{Hindawi}

Submit your manuscripts at

https://www.hindawi.com
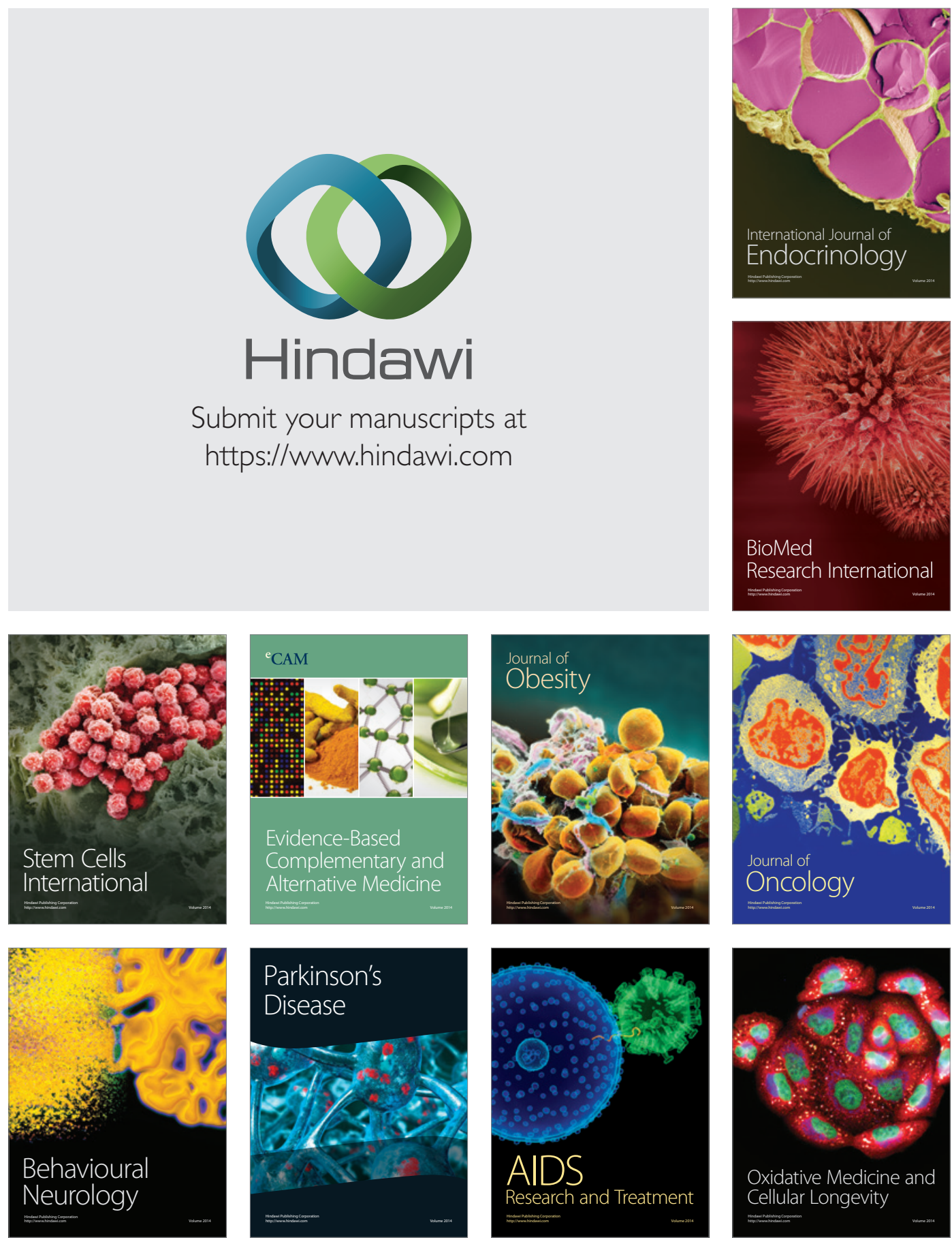